\title{
THE RIEMANN HYPOTHESIS FOR HILBERT SPACES OF ENTIRE FUNCTIONS
}

\author{
BY LOUIS DE BRANGES
}

\section{Contents}

\author{
History of the scattering method \\ Hilbert spaces of entire functions \\ Character zeta-functions \\ Hecke subgroups of the modular group \\ The Laplace-Beltrami operator \\ Existence of the scattering operator \\ Computation of the scattering operator \\ Spectral theory \\ The Riemann hypothesis
}

History of the scattering method. The scattering theory of linear differential operators is an approach to their spectral analysis which arises from the study of wave propagation. The medium in which waves are propagated is regarded as a linear system whose input comes from the remote past and whose output is given in the distant future. The scattering operator describes the energy-preserving transition from past to future.

The awareness of a relationship between scattering theory and that area of number theory associated with the Riemann hypothesis is at least twenty-five years old. It is found for example in an analysis of the Laplace-Beltrami operator by Ehrenpreis and Mautner [4] and in a congress address of Gelfand [6]. In the same years the author constructed the Hilbert spaces of entire functions which are used in the present formulation of scattering theory.

In 1972, Faddeev and Pavlov [5] applied the Lax-Phillips scattering theory to the Laplace-Beltrami operator, considered in a space of functions invariant under the action of the modular group. An account of their result in English, and a generalization, appeared in 1976 in a monograph by Lax and Phillips [7]. The important observation is made that the Riemann hypothesis is equivalent to decay properties in the wave propagation associated with the operator. But no geometric reason could be found for the propagation to have these

Address delivered at the winter meeting of the American Mathematical Society in Anaheim, California, January 1985; received by the editors October 15, 1985.

1980 Mathematics Subject Classification (1985 Revision). Primary 11M26, 47A40.

(C)1986 American Mathematical Society $0273-0979 / 86 \$ 1.00+\$ .25$ per page 
properties. Lax and Phillips clearly do not expect a proof of the Riemann hypothesis by these methods.

The formulation of scattering theory using the theory of Hilbert spaces of entire functions is more difficult than in the Lax-Phillips theory because there is more structure. Therefore it is understandable that the author's independent discovery in 1973 of results related to those of Faddeev and Pavlov obtained little attention $[2,3]$.

In the author's formulation, the scattering operator is described by a transfer function which is analytic in the complex plane instead of the unit disk or the upper half-plane. The reason for accepting the greater structure is that it is naturally present in connection with zeta-functions.

During the months of April, May, and June 1984, the author visited the Leningrad Branch of the V. A. Steklov Mathematical Institute under the exchange agreement between the National Academy of Sciences and the Academy of Sciences of the USSR. It was during this time that he became aware of a way of applying the additional structure in connection with the Riemann hypothesis.

The purpose of the present address is to describe this interesting development. An expository account is given of the theory of Hilbert spaces of entire functions as it applies to the Laplace-Beltrami operator. A conjecture is then made in this theory which implies the Riemann hypothesis.

Hilbert spaces of entire functions. The trouble with talking about Hilbert spaces of entire functions is that there is too much to say. An adequate motivation of the spaces will necessarily be very lengthy. Those who aim at new results in the field do not want to be caught in this familiar trap.

What does need to be said is that the theory of Hilbert spaces of entire functions is an invariant subspace theory for certain linear transformations in Hilbert space. The transformations are in general unbounded and partially defined. The meaning of "invariant subspace" needs to be clarified. An answer is provided by the theory.

This is a very nice invariant subspace theory because invariant subspaces always exist. There are enough for a spectral expansion of the transformation. And the natural invariant subspaces are totally ordered. Few other nontrivial examples of linear transformations in Hilbert space exist for which the invariant subspaces are known and are so usefully applied to the structure theory of the transformation.

The transformation is assumed given in a canonical model, in a form which is useful for study. If the transformation is not initially given in that form, then it is put in that form under a unitary equivalence which often appears as an eigenfunction expansion for a differential operator.

A terse way to introduce the relevant Hilbert spaces of entire functions is to characterize them by these axioms:

(H1) Whenever an element $F(z)$ of the space has a nonreal zero $w$, the function $F(z)(z-\bar{w}) /(z-w)$ belongs to the space and has the same norm as $F(z)$.

(H2) The linear functional defined on the space by $F(z)$ into $F(w)$ is continuous for every nonreal number $w$. 
(H3) The function $F^{*}(z)=\bar{F}(\bar{z})$ belongs to the space whenever $F(z)$ belongs to the space, and it always has the same norm as $F(z)$.

In connection with such a space, one thinks of a transformation, "multiplication by $z$ in the space," which takes $F(z)$ into $z F(z)$ whenever $F(z)$ and $z F(z)$ belong to the space. In the language of Stone and von Neumann, multiplication by $z$ is a closed symmetric transformation of deficiency index $(1,1)$ which is real with respect to a conjugation. The transformation is characterized by the fact that multiplication by $z-w$ has a bounded, partially defined inverse for every complex number $w$.

An example of such a space is obtained from any entire function $E(z)$ which satisfies the inequality

$$
|E(x-i y)|<|E(x+i y)|
$$

when $y>0$. Write $E(z)=A(z)-i B(z)$ where $A(z)$ and $B(z)$ are entire functions which are real for real $z$, and

$$
K(w, z)=[B(z) \bar{A}(w)-A(z) \bar{B}(w)] /[\pi(z-\bar{w})] .
$$

Define $\mathscr{H}(E)$ to be the set of entire functions $F(z)$ such that

$$
\|F\|^{2}=\int_{-\infty}^{+\infty}|F(t) / E(t)|^{2} d t
$$

is finite and such that the inequality

$$
|F(z)|^{2} \leqslant\|F\|^{2} K(z, z)
$$

holds for all complex $z$. Then $\mathscr{H}(E)$ is a Hilbert space of entire functions which satisfies the axioms $(\mathrm{H} 1),(\mathrm{H} 2)$, and $(\mathrm{H} 3)$. The expression $K(w, z)$ belongs to the space as a function of $z$ for every complex number $w$ and

$$
F(w)=\langle F(t), K(w, t)\rangle
$$

for every element $F(z)$ of the space.

Any Hilbert space of entire functions which satisfies $(\mathrm{H} 1),(\mathrm{H} 2)$, and $(\mathrm{H} 3)$ and contains a nonzero element is isometrically equal to a space $\mathscr{H}(E)$.

The referee remarks that the proof of this elementary theorem [1] is an instructive exercise for the reader.

Hint. Find the form of the reproducing kernel for the space.

Character zeta-functions. Let $r$ be a given positive integer. A character modulo $r$ is a function $\chi(n)$ of integers $n$, periodic of period $r$, which satisfies the identity $\chi(m n)=\chi(m) \chi(n)$ for all integers $m$ and $n$, and vanishes at points which are not relatively prime to $r$, but does not vanish identically. A character is an even or odd function. A character $\chi$ is said to be a primitive character modulo $r$ if it is a character modulo $r$ and if no character modulo $s$ exists, $s$ a proper divisor of $r$, which agrees with $\chi$ at points which are relatively prime to $r$. The principal character modulo $r$ is the unique character modulo $r$ whose values are zero and one. It is primitive only when $r=1$.

The zeta-function $\zeta_{\chi}(s)$ associated with a character $\chi$ modulo $r$ is defined by

$$
\zeta_{\chi}(s)=\sum_{n=1}^{\infty} \chi(n) n^{-s}
$$


The series is absolutely convergent in the half-plane $\operatorname{Re} s>1$ and defines an analytic function of $s$. The Euler product

$$
\zeta_{\chi}(s)=\prod \frac{1}{1-\chi(p) p^{-s}}
$$

which is taken over the primes $p$,implies that the zeta-function has no zeros in the half-plane.

Assume that $\chi$ is a primitive even character modulo $r$. Then a complex number $\varepsilon(\chi)$ of absolute value one exists such that the identity

$$
\varepsilon(\chi) \bar{\chi}(n)=\frac{1}{\sqrt{r}} \sum_{k=1}^{r} \chi(k) \cos \left(\frac{2 \pi n k}{r}\right)
$$

holds for every integer $n$. The theta-function $\theta_{\chi}(z)$ associated with $\chi$ is defined in the upper half-plane by

$$
\theta_{\chi}(z)=\sum_{-\infty}^{+\infty} \chi(n) \exp \left(\pi \operatorname{in}^{2} z / r\right) .
$$

The functional identity

$$
\theta_{\chi}(z)=\varepsilon(\chi) \sqrt{i / z} \theta_{\bar{\chi}}(-1 / z)
$$

is a consequence of the Poisson summation formula for the line. (The square root is taken with positive real part.) The functional identity for the corresponding zeta-functions

$$
\left(\frac{r}{\pi}\right)^{s / 2} \Gamma(s / 2) \zeta_{\chi}(s)=\varepsilon(\chi)\left(\frac{r}{\pi}\right)^{(1-s) / 2} \Gamma\left(\frac{1}{2}-\frac{1}{2} s\right) \zeta_{\bar{\chi}}(1-s)
$$

is obtained on passing to Mellin transforms. When $\chi$ is a nonprincipal character, each side is an entire function of $s$ which has constant phase on the line $\operatorname{Re} s=\frac{1}{2}$. When $\chi$ is the principal character, each side is analytic in the complex plane except for simple poles at the points $s=0$ and $s=1$.

The Riemann hypothesis for such a character zeta-function is the conjecture that the zeros of the function on the left (or right) are simple and lie on the line $\operatorname{Re} s=\frac{1}{2}$.

Note that Riemann stated the conjecture only for the principal character and that he did not require simplicity of zeros. The present statement is an addition of later authors, and there is no general agreement on this point. It represents the author's personal opinion about what is true and provable.

Hecke subgroups of the modular group. An analytic function of $z$ which maps the upper half-plane in a one-to-one fashion onto itself is of the form

$$
\frac{A z+B}{C z+D}
$$

for real numbers $A, B, C$, and $D$ such that $A D-B C=1$. Such mappings form a group under composition. The group, and certain of its subgroups of finite index, have long been recognized as relevant to the study of character zeta-functions.

If $r$ is a positive integer, define $\Gamma(r)$ to be the group of mappings of the upper half-plane onto itself which are of the form $z$ into $(A z+B) /(C z+D)$ for integers $A, B, C$, and $D$ such that $C$ is divisible by $r$ and $A D-B C=1$. 
Also of interest is the related group $\Gamma(r)^{*}$ generated by $\Gamma(r)$ and the mapping which takes $z$ into $-1 /(r z)$. When $r$ is not one, $\Gamma(r)$ is a normal subgroup of index two in $\Gamma(r)^{*}$.

The upper half-plane decomposes into equivalence classes under each group action. Points in the upper half-plane are considered equivalent with respect to one of these groups if one is obtained from the other under the action of the group.

Another kind of action is also considered. Points $z$ and $w$ in the upper half-plane are considered symmetric with respect to $\Gamma(r)^{*}$ if $z$ and $-\bar{w}$ are equivalent with respect to $\Gamma(r)^{*}$.

Examples of points which are self-symmetric with respect to $\Gamma(r)^{*}$ are points on any vertical line through a half-integer point on the real axis.

The action of $\Gamma(r)^{*}$ maps points in the upper half-plane which are not self-symmetric with respect to $\Gamma(r)^{*}$ into points which are not self-symmetric with respect to $\Gamma(r)^{*}$. The points in the upper half-plane which are not self-symmetric with respect to $\Gamma(r)^{*}$ form an open set whose complement has zero plane measure.

A fundamental region $\Omega(r)$ for $\Gamma(r)^{*}$ is a connected open subset of the upper half-plane, which contains no distinct equivalent points with respect to $\Gamma(r)^{*}$, such that the points of the upper half-plane which are equivalent with respect to $\Gamma(r)^{*}$ to no element of $\Omega(r)$ form a set of zero plane measure.

A fundamental region for $\Gamma(r)^{*}$ always exists. Enough of the construction of such a region will be given to determine what elements of the region are self-symmetric with respect to $\Gamma(r)^{*}$.

A point in the upper half-plane is said to be admissible with respect to $\Gamma(r)^{*}$ if all points of the same $\Gamma(r)^{*}$-equivalence class which lie at a maximum distance from the real axis differ from each other by integers. The admissible points with respect to $\Gamma(r)^{*}$ form an open set whose complement has zero plane measure. The action of $\Gamma(r)^{*}$ maps admissible points with respect to $\Gamma(r)^{*}$ into admissible points with respect to $\Gamma(r)^{*}$.

Consider the set of admissible points which are not self-symmetric with respect to $\Gamma(r)^{*}$. It is an open subset of the upper half-plane whose complement has zero plane measure. The set is invariant under the action of $\Gamma(r)^{*}$, and it is the union of its connected components. The action of $\Gamma(r)^{*}$ maps each component onto a component. Two components are considered equivalent if they are mapped into each other under the action of $\Gamma(r)^{*}$.

Certain components are easily identified. A component is said to be distinguished if its elements lie at a maximum distance from the real axis within their equivalence classes. Each component is equivalent to a distinguished component. Each distinguished component has, as part of its boundary, two vertical half-lines, one-half unit apart, above half-integer points on the real axis.

There are two equivalence classes of components because there are two equivalence classes of distinguished components. Two distinguished components are considered adjacent if their closures have a nonempty intersection. The intersection is then a vertical half-line. Adjacent distinguished components are never equivalent. 
A fundamental region for $\Gamma(r)^{*}$ is obtained as the interior of the union of the closures of two adjacent distinguished components. The self-symmetric elements of the fundamental region are those which belong to the intersection of the closures of the two components.

A fundamental region for $\Gamma(r)$ is obtained by a similar piecing together of two adjacent fundamental regions $\Gamma(r)^{*}$.

The Laplace-Beltrami operator. The analytic one-to-one mappings of the upper half-plane onto itself are isometries of the upper half-plane, considered with its hyperbolic geometry. The Laplace-Beltrami operator is the second-order partial differential operator which appears as the Laplacian operator for this hyperbolic geometry. It commutes with the group action. Its spectral theory appears in the Bargmann decomposition theory of unitary representations of the group into irreducible representations. And it also appears in a different way in connection with the action of Hecke subgroups of the modular group.

The Laplace-Beltrami operator is formally defined to take $F(z)$ into $G(z)$ where

$$
G(z)=(z-\bar{z})^{2} \frac{\partial^{2} F}{\partial z \partial \bar{z}}
$$

The differential operator can be considered in various Hilbert spaces in which it is selfadjoint. Some of these spaces will now be constructed and relations between them will be found.

If $\chi$ is an even character modulo $r$, define $\mathscr{P}_{\chi}(r)$ to be the Hilbert space of equivalence classes of measurable functions $F(z)$ of $z$ in the upper half-plane such that

$$
F(z)=\chi(D) F\left(\frac{A z+B}{C z+D}\right)
$$

for all integers $A, B, C$, and $D$ with $C$ divisible by $r$ and $A D-B C=1$, and such that

$$
\|F\|_{\mathscr{P}_{x}(r)}^{2}=\iint_{\Omega}|F(z)|^{2} y^{-2} d x d y
$$

is finite, where $\Omega$ is a fundamental region for $\Gamma(r)$. The integral does not depend on the choice of fundamental region.

Also consider the Hilbert space $\mathscr{P}(\infty)$ of equivalence classes of measurable functions $F(z)$ of $z$ in the upper half-plane such that $F(z-t)=F(z)$ for all real $t$ and such that

$$
\|F\|_{\mathscr{P}(\infty)}^{2}=\int_{0}^{\infty}|F(i y)|^{2} y^{-2} d y
$$

is finite.

The action of the Laplace-Beltrami operator in the space $\mathscr{P}(\infty)$ reduces to a question in Fourier analysis on the multiplicative group of the positive half-line. The operator becomes an ordinary differential operator in this space. It is selfadjoint and has an absolutely continuous spectrum.

The action of the Laplace-Beltrami operator in the space $\mathscr{P}_{\chi}(r)$ is considerably more complicated. Information about the operator is obtained from a 
summation isometry which commutes with the operator. The isometric property of summation holds only for nonprincipal characters. Otherwise the summation transformation is only a partial isometry.

THEOREM 1. Assume that $\chi$ is a nonprincipal even character modulo $r$. Then an isometric transformation exists of the space $\mathscr{P}(\infty)$ into the space $\mathscr{P}_{\chi}(r)$ which takes $F(z)$ into

$$
\frac{1}{2} \sum \chi(D) F\left(\frac{A z+B}{C z+D}\right)
$$

where summation is over all pairs of relatively prime integers $C$ and $D$ such that $C$ is divisible by $r, A$ and $B$ being corresponding integers such that $A D-B C=1$. The adjoint transformation of $\mathscr{P}_{\chi}(r)$ into $\mathscr{P}(\infty)$ is a partial isometry which takes $F(z)$ into

$$
\int_{0}^{1} F(z-t) d t
$$

The summation which is used to define the isometry need not be absolutely convergent. But it is absolutely convergent almost everywhere under the hypothesis that

$$
\int_{0}^{\infty}|F(i y)| y^{-2} d y
$$

is finite. The transformation is otherwise defined by continuity in the Hilbert space norms.

Because of the summation isometry, a part of the spectrum of the LaplaceBeltrami operator in $\mathscr{P}_{\chi}(r)$ is subject to an elementary spectral analysis. This is the part of the spectrum which lies in the range of the summation isometry. There is also a part of the spectrum about which the summation isometry gives no information. This is the part of the spectrum which lies orthogonal to the range of the summation isometry. This reducing subspace for the LaplaceBeltrami operator in $\mathscr{P}_{\chi}(r)$ is the set of elements $F(z)$ of the space such that $\int_{0}^{1} F(z-t) d t=0$ almost everywhere. The restriction of the Laplace-Beltrami operator to the orthogonal complement of this subspace has absolutely continuous spectrum.

Proof of Theorem 1. Consider any measurable function $F(z)$ of $z$ in the upper half-plane such that $F(z-t)=F(z)$ for all real numbers $t$ and such that $\int_{0}^{\infty}|F(i y)| y^{-2} d y$ is finite. Then the sum

$$
G(z)=\frac{1}{2} \sum \chi(D) F\left(\frac{A z+B}{C z+D}\right)
$$

is absolutely convergent almost everywhere and the inequality

$$
\begin{aligned}
& \iint_{\Omega}|G(z)| y^{-2} d x d y \\
& \quad \leqslant \frac{1}{2} \sum \iint_{\Omega}\left|F\left(\frac{A z+B}{C z+D}\right)\right| y^{-2} d x d y=\int_{0}^{\infty}|F(i y)| y^{-2} d y
\end{aligned}
$$

is satisfied. 
The notation $\Omega$ is used for a fundamental region for the group $\Gamma(r)$. The last identity is due to the fact that, for suitable choices of $A$ and $B$, the region $\Omega$ is the image of a subregion of the half-strip $-\frac{1}{2}<\operatorname{Re} z<\frac{1}{2}$ under the transformation which takes $z$ into $(A z+B) /(C z+D)$. These subregions are disjoint and essentially fill the portion of the half-strip which lies above the real axis.

The principal step in the proof of the theorem is the verification of the identity

$$
F(z)=\int_{0}^{1} G(z-t) d t .
$$

This is done by a term-by-term integration of the sum defining $G(z)$, which is permissible by the previous discussion of its convergence. It is sufficient to show that

$$
\sum \int_{0}^{1} \chi(D) F\left(\frac{A z-A t+B}{C z-C t+D}\right) d t=0
$$

whenever $C$ is a positive integer which is divisible by $r$ and summation is over the integers $D$ which are relatively prime to $r, A$ and $B$ being corresponding integers such that $A D-B C=1$. Indeed the sum is equal to

$$
\sum \chi(n) \int_{-\infty}^{+\infty} F\left(\frac{i y}{|C z|^{2}}\right) d x,
$$

where summation is over the integers $n$ which are relatively prime to $r$. The sum is zero because $\chi$ is assumed to be a nonprincipal character modulo $r$.

The isometric property of the summation is now obtained by the same decomposition of the upper half of the strip $-\frac{1}{2}<\operatorname{Re} z<\frac{1}{2}$. This gives the identity

$$
\begin{aligned}
\int_{0}^{\infty}|F(i y)|^{2} y^{-2} d y & =\int_{0}^{\infty} \int_{0}^{1} F(z) \bar{G}(z) y^{-2} d x d y \\
& =\frac{1}{2} \sum \chi(D) \iint_{\Omega} F\left(\frac{A z+B}{C z+D}\right) \bar{G}(z) y^{-2} d x d y \\
& =\iint_{\Omega} \frac{1}{2} \sum \chi(D) F\left(\frac{A z+B}{C z+D}\right) \bar{G}(z) y^{-2} d x d y \\
& =\iint_{\Omega}|G(z)|^{2} y^{-2} d x d y
\end{aligned}
$$

The computation of the adjoint of the summation transformation is now made by a similar argument. Assume that $F(z)$ and $G(z)$ are as above with $F(z)$ in $\mathscr{P}(\infty)$ and $G(z)$ in $\mathscr{P}_{\chi}(r)$. Then the identity

$$
\int_{0}^{\infty} \int_{0}^{1} F(z) \bar{H}(z) y^{-2} d x d y=\iint_{\Omega} G(z) \bar{H}(z) y^{-2} d x d y
$$

holds for every element $H(z)$ of $\mathscr{P}_{\chi}(r)$. This completes the proof of the theorem, since the left side can be written

$$
\int_{0}^{\infty} F(i y) \int_{0}^{1} \bar{H}(i y-t) d t y^{-2} d y \text {. }
$$


Existence of the scattering operator. The transformation which takes $F(z)$ into $F(-1 / r z)$ is an isometry of $\mathscr{P}_{\chi}(r)$ into $\mathscr{P}_{\bar{\chi}}(r)$. The transformation is well behaved with respect to the ranges of the summation isometries if $\chi$ is a primitive character modulo $r$.

THEOREM 2. Assume that $\chi$ is a primitive even character modulo $r, r$ not one. If $F(z)$ belongs to $\mathscr{P}(\infty)$, then a unique element $G(z)$ of $\mathscr{P}(\infty)$ exists such that

$$
\frac{1}{2} \sum \bar{\chi}(D) G\left(\frac{A z+B}{C z+D}\right)=\frac{1}{2} \sum \chi(D) F\left(\frac{A-B r z}{C-D r z}\right),
$$

where summation is over all pairs of relatively prime integers $C$ and $D$ such that $C$ is divisible by $r, A$ and $B$ being corresponding integers such that $A D-B C=1$. The identity

$$
\begin{gathered}
\varepsilon(x) r^{i x / 2}\left(\frac{r}{\pi}\right)^{(1+i x) / 2} \Gamma((1+i x) / 2) \zeta_{\bar{\chi}}(1+i x) \int_{0}^{\infty} F(i t) t^{(i x-3) / 2} d t \\
=\left(\frac{r}{\pi}\right)^{(1-i x) / 2} \Gamma((1-i x) / 2) \zeta_{\chi}(1-i x) \int_{0}^{\infty} G(i t) t^{(-i x-3) / 2} d t
\end{gathered}
$$

holds for almost all real $x$.

Proof of Theorem 2. The proof is an application of the Poisson summation formula in the plane. Use is made of the identity

$$
\begin{gathered}
\int_{-\infty}^{+\infty} \int_{-\infty}^{+\infty} \exp \left(\frac{\pi a^{2}|u z+v|^{2}}{y}\right) \exp (2 \pi i \xi u+2 \pi i \eta v) d u d v \\
=\frac{1}{a^{2}} \exp \left(-\frac{\pi|\eta z-\xi|^{2}}{a^{2} y}\right),
\end{gathered}
$$

which holds for all real numbers $\xi$ and $\eta$ when $a>0$ and $z=x+i y$ is in the upper half-plane. By the Poisson summation formula,

$$
\begin{aligned}
& \sum_{-\infty}^{+\infty} \sum_{-\infty}^{+\infty} f(m, n) \exp \left(-\frac{\pi a^{2}|m z+n|^{2}}{r y}\right) \\
& \quad=\sum_{-\infty}^{+\infty} \sum_{-\infty}^{+\infty} g(m, n) \frac{1}{a^{2}} \exp \left(-\frac{\pi|m z+n|^{2}}{r a^{2} y}\right)
\end{aligned}
$$

whenever $f(m, n)$ and $g(m, n)$ are functions of integers $m$ and $n$ modulo $r$ such that

$$
r g(m, n)=\sum_{u=1}^{r} \sum_{v=1}^{r} f(u, v) \exp (2 \pi i m u+2 \pi i n v) .
$$

The identity is used with $f(m, n)=\chi(n) \sqrt{r}$ when $m$ is divisible by $r$ and $f(m, n)=0$ otherwise. Then $g(m, n)=\varepsilon(\chi) \bar{\chi}(m)$.

Fundamental examples of functions $F(z)$ and $G(z)$ which satisfy the hypotheses of the theorem are given by

$$
F(x+i y)=\theta_{\chi}\left(\frac{i a^{2}}{y}\right)
$$


and

$$
G(x+i y)=\varepsilon(x) \frac{1}{a^{2} \sqrt{r}} \theta_{\bar{x}}\left(\frac{i}{a^{2} r y}\right)
$$

for a positive number $a$. They belong to $\mathscr{P}(\infty)$ because of the functional identity for the theta-function. The previous application of the Poisson summation formula in the plane shows that

$$
\begin{aligned}
\frac{1}{2} \sum \chi & (D) F\left(\frac{A z+B}{C z+D}\right)=\frac{1}{2} \sum_{-\infty}^{+\infty} \sum_{-\infty}^{+\infty} \chi(n) \exp \left(-\frac{\pi a^{2}|r m z+n|^{2}}{r y}\right) \\
& =\frac{1}{2} \sum_{-\infty}^{+\infty} \sum_{-\infty}^{+\infty} \varepsilon(\chi) \bar{\chi}(m) \frac{1}{a^{2} \sqrt{r}} \exp \left(-\frac{\pi|m z+n|^{2}}{a^{2} r y}\right) \\
& =\frac{1}{2} \sum \bar{\chi}(D) G\left(\frac{A-B r z}{C-D r z}\right) .
\end{aligned}
$$

The desired identity follows since

$$
\int_{0}^{\infty} F(i t) t^{(i x-3) / 2} d t=2 a^{-1+i x}\left(\frac{r}{\pi}\right)^{(1-i x) / 2} \Gamma((1-i x) / 2) \zeta_{\chi}(1-i x)
$$

and

$$
\begin{aligned}
\int_{0}^{\infty} & G(i t) t^{(i x-3) / 2} d t \\
& =2 a^{-1-i x} r^{-i x / 2} \varepsilon(x)\left(\frac{r}{\pi}\right)^{(1-i x) / 2} \Gamma((1-i x) / 2) \zeta_{\bar{\chi}}(1-i x)
\end{aligned}
$$

The theorem now follows by linearity and continuity since the finite linear combinations of such special functions are dense in $\mathscr{P}(\infty)$.

The transformation constructed in Theorem 2 has a geometric interpretation. Waves are propagated through a medium. At first the waves flow uniformly downwards along vertical lines. This propagation is made using the Laplace-Beltrami operator in $\mathscr{P}(\infty)$. Then the waves enter a closed box against whose walls they are reflected. This propagation is made using the LaplaceBeltrami operator in $\mathscr{P}_{\chi}(r)$. Then the waves come out of the box and flow uniformly upwards on vertical lines. This propagation is again made using the Laplace-Beltrami operator in $\mathscr{P}(\infty)$.

The transformation constructed in Theorem 2 describes the transition from the initial downward flow to the final upward flow.

Computation of the scattering operator. A computation of the transformation of Theorem 2 is easily made. The notation $\varphi(r)$ is used for the number of integers modulo $r$ which are relatively prime to $r$.

THEOREM 3. Assume that $\chi$ is a primitive even character modulo $r, r$ not one. For positive numbers $t$, define

$$
k_{\chi}(t)=\sum \frac{\varphi(n) \chi(n)}{n} \frac{1}{\sqrt{t-n^{2}}},
$$


where summation is over the positive integers $n$ such that $n^{2}<t$. Let $F(z)$ and $G(z)$ be elements of $\mathscr{P}(\infty)$ such that

$$
\frac{1}{2} \sum \bar{\chi}(D) G\left(\frac{A z+B}{C z+D}\right)=\frac{1}{2} \sum \chi(D) F\left(\frac{A-B r z}{C-D r z}\right),
$$

where summation is over all pairs of relatively prime integers $C$ and $D$ such that $C$ is divisible by $r, A$ and $B$ being corresponding integers such that $A D-B C=1$. Then

$$
G(i y)=\int_{1}^{\infty} F\left(\frac{i}{y r t}\right) y k_{\chi}(t) d t .
$$

The integral is interpreted as a limit in the mean square sense of $\int_{1}^{a}$.

Proof of TheOrem 3. By the interpretation of the integral, it is sufficient to establish the identity in the case that $F(i y)$ vanishes for small $y$. In this case the integral

$$
\int_{0}^{\infty}|F(i y)| y^{-2} d y
$$

is finite. As in the proof of Theorem 1, the condition allows the interchange of summation and integration which follows. By Theorem 1,

$$
\begin{aligned}
G(i y) & =\int_{0}^{1} \frac{1}{2} \sum \chi(D) F\left(\frac{A-B r z+B r t}{C-D r z+D r t}\right) d t \\
& =\frac{1}{2} \sum \chi(D) \int_{0}^{1} F\left(\frac{A-B r z+B r t}{C-D r z+D r t}\right) d t \\
& =\sum_{n=1}^{\infty} \frac{\varphi(n) \chi(n)}{n} \int_{-\infty}^{+\infty} F\left(\frac{i r y}{r^{2} t^{2}+r^{2} n^{2} y^{2}}\right) d t \\
& =\sum_{n=1}^{\infty} \frac{\varphi(n) \chi(n)}{n} \int_{n^{2}}^{\infty} F\left(\frac{i}{t r y}\right) \frac{y}{\sqrt{t-n^{2}}} d t \\
& =\int_{1}^{\infty} F\left(\frac{i}{t r y}\right) y k_{\chi}(t) d t .
\end{aligned}
$$

The last interchange of summation and integration is possible because $F(i y)$ vanishes for small $y$. This makes the sum contain only a finite number of nonzero terms. The theorem follows.

An estimate of Mellin transforms results.

THEOREM 4. Assume that $\chi$ is a primitive even character modulo $r, r$ not one. Then

$$
W_{\chi}(z)=r^{-1 / 2} \int_{1}^{\infty} k_{\chi}(t) t^{(i z-1) / 2} d t
$$


is analytic and bounded by one in the upper half-plane. The identity

$$
\begin{aligned}
& \left(\frac{r}{\pi}\right)^{(1-i z) / 2} \Gamma((1-i z) / 2) \zeta_{\chi}(1-i z) W_{\chi}(z) \\
& \quad=\varepsilon(\chi)\left(\frac{r}{\pi}\right)^{(1+i z) / 2} \Gamma((1+i z) / 2) \zeta_{\bar{\chi}}(1+i z)
\end{aligned}
$$

holds in the upper half-plane.

Proof of TheOrem 4. If $F(z)$ and $G(z)$ are elements of $\mathscr{P}(\infty)$ such that

$$
G(i y)=\int_{1}^{\infty} F\left(\frac{i}{t r y}\right) y k_{\chi}(t) d t,
$$

then the identity

$$
\begin{aligned}
\int_{0}^{\infty} & G(i t) t^{(-i z-3) / 2} d t \\
& =r^{i z / 2} W_{\chi}(z) \int_{0}^{\infty} F(i t) t^{(i z-3) / 2} d t
\end{aligned}
$$

holds for almost all real $z=x$ by the mean square theory of the Mellin transformation. The identity given in the statement of the theorem now follows for almost all real $z=x$ by the identity in the statement of Theorem 2. Since the transformation which takes $F(z)$ into $G(z)$ is isometric in $\mathscr{P}(\infty)$ by Theorems 2 and 3, the function $W_{\chi}(z)$ has absolute value one almost everywhere on the real axis by the mean square theory of the Mellin transformation. Since $W_{\chi}(z)$ is the Mellin transform of a function which vanishes in the unit interval, $W_{\chi}(z)$ is the boundary value function of a function which is analytic and bounded by one in the upper half-plane and satisfies the same identities in the half-plane. This completes the proof of the theorem.

Spectral theory. Since $W_{\chi}(z)$ is not a constant of absolute value one, the strict inequality

$$
\left|W_{\chi}(z)\right|<1
$$

holds in the upper half-plane. When $a \geqslant 1$, define

$$
E_{\chi}(a, z)=a^{-i z / 2}\left(\frac{r}{\pi}\right)^{(1-i z) / 2} \Gamma((1-i z) / 2) \zeta_{\chi}(1-i z) .
$$

Since

$$
\left|E_{\chi}(a, x-i y)\right|<\left|E_{\chi}(a, x+i y)\right|
$$

when $y>0$, a space $\mathscr{H}\left(E_{\chi}(a)\right)$ exists. The space appears in a precise description of the spectral properties of the Laplace-Beltrami operator.

THEOREM 5. Assume that $\chi$ is a primitive even character modulo $r, r$ not one, and that $a \geqslant 1$. Let $F(z)$ and $G(z)$ be elements of $\mathscr{P}(\infty)$, which vanish for $y>a r^{-1 / 2}$, such that

$$
\frac{1}{2} \sum \bar{\chi}(D) G\left(\frac{A z+B}{C z+D}\right)=\frac{1}{2} \sum \chi(D) F\left(\frac{A-B r z}{C-D r z}\right)
$$


where summation is over all pairs of relatively prime integers $C$ and $D$ such that $C$ is divisible by $r, A$ and $B$ being corresponding integers such that $A D-B C=1$. Then a corresponding element $H(z)$ of $\mathscr{H}\left(E_{\bar{\chi}}(a)\right)$ exists such that the identities

$$
H(z)=E_{\bar{\chi}}(1, z) \int_{0}^{a r^{-1 / 2}} F(i t) t^{(-i z-3) / 2} d t
$$

and

$$
H(-z)=\bar{\varepsilon}(\chi) E_{\chi}(1, z) \int_{0}^{a r^{-1 / 2}} G(i t) t^{(-i z-3) / 2} d t
$$

hold when $y>0$. The identities

$$
4 \pi\|F\|_{\mathscr{P}(\infty)}^{2}=\|H\|_{\mathscr{H}\left(E_{\overline{\mathrm{x}}}(a)\right)}^{2}=4 \pi\|G\|_{\mathscr{P}(\infty)}^{2}
$$

are satisfied. Every element of $\mathscr{H}\left(E_{\bar{\chi}}(a)\right)$ is equal to such a function $H(z)$ for unique such elements $F(z)$ and $G(z)$ of $\mathscr{P}(\infty)$.

Proof of Theorem 5. The proof is another application of the mean square theory of the Mellin transformation. Consider the transformation which takes $F(z)$ into

$$
f(z)=\int_{0}^{a r^{-1 / 2}} F(i t) t^{(-i z-3) / 2} d t
$$

when $F(z)$ is an element of $\mathscr{P}(\infty)$ which vanishes for $y>a r^{-1 / 2}$. The set of such functions $f(z)$ is the set $\mathscr{M}\left(a r^{-1 / 2}\right)$ of functions $f(z)$ which are analytic and of bounded type in the upper half-plane, with square integrable boundary value on the real axis, such that the inequality

$$
|f(x+i y)|^{2} \leqslant \frac{a^{y} r^{-y / 2}}{4 \pi y} \int_{-\infty}^{+\infty}|f(t)|^{2} d t
$$

holds in the upper half-plane.

Consider an element $F(z)$ of $\mathscr{P}(\infty)$ such that the corresponding element $G(z)$, defined as in Theorem 2, vanishes when $y<a r^{-1 / 2}$. Then the identity

$$
\int_{0}^{a r^{-1 / 2}} F(i t) t^{(-i z-3) / 2} d t=r^{i z / 2} W_{\bar{\chi}}(z) \int_{a r^{-1 / 2}}^{\infty} G(i t) t^{(i z-3) / 2} d t
$$

holds in the upper half-plane. The set of functions $g(z)$ which are of the form

$$
g(z) \int_{a r^{-1 / 2}}^{\infty} G(i t) t^{(i z-3) / 2} d t
$$

for such a function $G(z)$ is the set $\mathscr{M}\left(a^{-1} r^{1 / 2}\right)$ of functions $g(z)$, analytic and of bounded type in the upper half-plane, which have square integrable boundary values on the real axis, such that the inequality

$$
|g(z+i y)|^{2} \leqslant \frac{a^{-y} r^{y / 2}}{4 \pi y} \int_{-\infty}^{+\infty}|g(t)|^{2} d t
$$

holds in the upper half-plane.

The spaces $\mathscr{M}\left(a r^{-1 / 2}\right)$ and $\mathscr{M}\left(a^{-1} r^{1 / 2}\right)$ are considered in the metric of $L^{2}(-\infty,+\infty)$ for the boundary value functions on the real axis. Multiplication by $r^{i z / 2} W_{\bar{\chi}}(z)$ is an isometric transformation of $\mathscr{M}\left(a^{-1} r^{1 / 2}\right)$ into $\mathscr{M}\left(a r^{1 / 2}\right)$. The orthogonal complement of the range of the isometry is a Hilbert space of 
functions which are analytic in the upper half-plane. The theorem follows because multiplication by

$$
\left(\frac{r}{\pi}\right)^{(1-i z) / 2} \Gamma((1-i z) / 2) \zeta_{\bar{\chi}}(1-i z)
$$

is an isometric transformation of the space onto $\mathscr{H}\left(E_{\bar{\chi}}(a)\right)$.

The Riemann hypothesis. This expansion in the theory of Hilbert spaces of entire functions is similar to the result of Faddeev and Pavlov, but is obtained by a different method. The issue of the Riemann hypothesis is a natural one to raise in the formulation of the expansion using Hilbert spaces of entire functions.

In the present expansion, special spaces $\mathscr{H}(E)$ appear for which $E(z-i)$ and $E^{*}(z)$ are linearly dependent. The existence of such a linear relation is equivalent to the functional identity for the character zeta-functions which appear in that context.

An equivalent statement of the condition is that $E(z)$ has constant phase on the line $\bar{z}=z+i$. The issue of the Riemann hypothesis is the question of whether the zeros of $E(z)$ are simple and lie on the line. A similar question can be asked for any space $\mathscr{H}(E)$ such that $E(z-i)$ and $E^{*}(z)$ are linearly dependent.

The answer in general is no. If $E(z-i)$ and $E^{*}(z)$ are linearly dependent for a space $\mathscr{H}(E)$, then it is not necessarily true that the zeros of $E(z)$ are simple and lie on the desired line. All that can be said is that the zeros of $E(z)$ lie in the strip $-1 \leqslant y \leqslant 0$ and that they are symmetrically placed about the line through the center of the strip.

To see this, it is sufficient to consider examples in which $E(z)$ is a polynomial. The condition that a space $\mathscr{H}(E)$ exists is that $E(z)$ has no zeros above the real axis and at least one zero below the axis. The condition that $E(z-i)$ and $E^{*}(z)$ are linearly dependent is the condition that the zeros of $E(z)$ are symmetrically placed about the line $y=-\frac{1}{2}$. The zeros are not subject to any other constraint. No analogue of the Riemann hypothesis is true when no further condition is imposed.

The question then arises as to what this additional condition should be. The answer which is now given is a condition which does imply that the zeros lie where they are wanted. But it is a stronger condition. It is possible for the zeros to lie on the desired line and to be simple without having the condition satisfied. Nevertheless the condition is a natural one to impose for the desired purpose. No weaker condition of the same kind seems to be available.

A statement of the condition will now be made, and it will be shown that it has the desired implications.

THEOREM 6. Assume that $\mathscr{H}(E)$ is a given space such that $E(z-i)$ and $E^{*}(z)$ are linearly dependent. If the real part $\langle F(t+i), F(t)\rangle$ is nonnegative whenever $F(z)$ is an element of the space such that $F(z+i)$ belongs to the space, then every zero $w$ of $E(z)$ such that neither $w$ nor $w+i$ is real, and such that $E(w+i)$ is not equal to $E(w-i)$, is simple and lies on the line $\bar{w}=w+i$. 
Proof of Theorem 6. Note that

$$
K(w, z)=\frac{E(z) \bar{E}(w)-E^{*}(z) E(\bar{w})}{2 \pi i(\bar{w}-z)}
$$

for any space $\mathscr{H}(E)$. The identity has interesting consequences when $E(z-i)$ and $E^{*}(z)$ are linearly dependent. If $w$ is a zero of $E(z)$, then the identity

$$
\bar{E}(w+i) K(w, z+i)=-\bar{E}(w-i) K(w+i, z)
$$

is satisfied. When $w$ is not real, $K(w, z)$ cannot be identically zero and $E(w-i)$ is nonzero. When $w+i$ is not real, $K(w+i, z)$ cannot be identically zero and $E(w+i)$ is nonzero. When $w$ is such a zero of $E(z)$, the identity produces a nontrivial element $F(z)$ of the space such that $F(z+i)$ belongs to the space. If $F(z)$ is any element of the space such that $F(z+i)$ belongs to the space, then

$$
\begin{aligned}
\langle F(t+i), K(w, t)\rangle & +\langle F(t), K(w, t+i)\rangle \\
= & \frac{E(w+i)-E(w-i)}{E(w+i)} F(w+i) .
\end{aligned}
$$

The inner product is taken in the metric of $\mathscr{H}(E)$.

For the interpretation of the identity, consider the scalar product defined by

$$
\langle F(t+i), G(t)\rangle+\langle F(t), G(t+i)\rangle
$$

on elements $F(z)$ and $G(z)$ of the space such that $F(z+i)$ and $G(z+i)$ belong to the space. Then the functions of the form $K(w, z)$, where $w$ is a zero of $E(z)$, form an orthogonal set with respect to the scalar product. This is true since $K(w, z+i)$ vanishes at the zeros of $E(z)$ other than $\bar{w}-i$ if $w$ is a zero of $E(z)$. If, on the other hand, $w$ is a zero of $E(z)$ such that $w=\bar{w}-i$, then the identities

$$
K(w, w+i)=\frac{E^{\prime}(w) \bar{E}(w-i)}{2 \pi i}
$$

and

$$
K(w+i, w)=-\frac{E^{\prime}(w) \bar{E}(w+i)}{2 \pi i}
$$

are satisfied. They imply the identity

$$
K(w, w+i)+K(w+i, w)=E^{\prime}(w) \frac{\bar{E}(w-i)-\bar{E}(w+i)}{2 \pi i} .
$$

Because of these identities, it becomes interesting to make use of the hypothesis that the scalar product is nonnegative. By hypothesis, the expression

$$
\begin{aligned}
& \langle K(w, t+i), K(w, t)\rangle+\langle K(w, t), K(w, t+i)\rangle \\
& \quad=K(w, w+i)+K(w+i, w)
\end{aligned}
$$

is nonnegative. If it is possible, then it follows immediately that $w=\bar{w}-i$ and that $E^{\prime}(w)$ is nonzero. In this case, $w$ is a simple zero of $E(z)$ which lies on the desired line and $E(w+i)$ is not equal to $E(w-i)$. 
It remains to consider the possibility that the last expression is zero. By the Schwarz inequality, the expression

$$
\langle F(t+i), K(w, t)\rangle+\langle F(t), K(w, t+i)\rangle
$$

is zero for every element $F(z)$ of the space such that $F(z+i)$ belongs to the space. By the above computation of the expression, the quantity

$$
[E(w+i)-E(w-i)] F(w+i)
$$

vanishes for every element $F(z)$ of the space such that $F(z+i)$ belongs to the space. Since $E(w+i)$ is not equal to $E(w-i)$ by hypothesis, $F(w+i)$ vanishes for every element $F(z)$ of the space such that $F(z+i)$ belongs to the space.

Since neither $w$ nor $w+i$ is real by hypothesis, $F(z) /(z-w-i)$ is again such an element of the space whenever $F(z)$ is such an element of the space. A contradiction is obtained since iteration produces an element $F(z)$ of the space such that $F(z+i)$ belongs to the space but such that $F(w+i)$ is nonzero. This completes the proof of the theorem.

If the stronger hypothesis is made that the real part of $\langle F(t+i), F(t)\rangle$ is positive whenever $F(z)$ is a nonzero element of the space such that $F(z+i)$ belongs to the space, then $E(z)$ has no zero $w$ such that $w$ or $w+i$ is real or such that $E(w+i)=E(w-i)$. This stronger hypothesis holds if sufficiently many zeros of $E(z)$ are simple and lie on the desired line.

THEOREM 7. Assume that $\mathscr{H}(E)$ is a given space such that $E(z-i)$ and $E^{*}(z)$ are linearly dependent and such that the real part of $\langle F(t+i), F(t)\rangle$ is nonnegative whenever $F(z)$ is an element of the space such that $F(z+i)$ belongs to the space. If every element of the space belongs to the closed span of the functions of the form

$$
\frac{E(z)}{z-w}
$$

where $w$ is a zero of $E(z)$ such that neither $w$ nor $w+i$ is real and such that $E(w+i)$ is not equal to $E(w-i)$, then the real part of $\langle F(t+i), F(t)\rangle$ is positive whenever $F(z)$ is a nonzero element of the space such that $F(z+i)$ belongs to the space.

Proof of Theorem 7. Assume that $F(z)$ is an element of space such that the real part of $\langle F(t+i), F(t)\rangle$ is zero. If $w$ is a zero of $E(z)$ such that neither $w$ nor $w+i$ is real and such that $E(w+i)$ is not equal to $E(w-i)$, then $F(w+i)$ vanishes by the proof of Theorem 6 . Thus $F(z)$ is orthogonal in the metric of $\mathscr{H}(E)$ to

$$
K(w+i, z)=\frac{E(z) \bar{E}(w+i)}{2 \pi i(w-z)},
$$

where $E(w+i)$ is nonzero because $w+i$ lies in the upper half-plane. Since the space is assumed to be the closed span of such functions, $F(z)$ vanishes identically. This completes the proof of the theorem. 
The hypotheses of Theorems 6 and 7 are conjectured to apply to the function $E_{\chi}(a, z)$ when $\chi$ is a primitive even character modulo $r, r$ not one, and $a=1$.

\section{REFERENCES}

1. L. de Branges, Espaces Hilbertiens de Fonctions Entières, Masson, Paris, 1972.

2. __ Modular spaces of entire functions, J. Math. Anal. Appl. 44 (1973), 192-205.

3. Coefficients of modular forms, J. Math. Anal. Appl. 45 (1974), 300-323.

4. L. Ehrenpreis and F. J. Mautner, Some properties of the Fourier-transform on semisimple Lie Groups. III, Trans. Amer. Math. Soc. 90 (1959), 431-484.

5. L. D. Faddeev and B. S. Pavlov, Scattering theory and automorphic functions, Boundary Value Problems of Mathematical Physics and Related Questions in the Theory of Functions, 6, Zap. Naučn. Sem. Leningrad. Otdel. Mat. Inst. Steklov (LOMI) 27 (1972), 161-193. (Russian) Mathematical Reviews 47 \# 9315.

6. I. M. Gelfand, Automorphic functions and the theory of representations, Proc. Internat. Congress of Mathematicians, Stockholm 1962, pp. 74-85.

7. P. D. Lax and R. S. Phillips, Scattering theory and automorphic functions, Princeton Univ. Press, Princeton, 1976.

Department of Mathematics, Purdue University, Lafayette, Indiana 47907 
